\title{
Associated impact of inorganic fertilizers and pesticides on microbial communities in soils
}

\author{
Jian Chen $\cdot$ Xuliang Zhuang $\cdot$ Huijun Xie · \\ Zhihui Bai $\cdot$ Hongyan Qi $\cdot$ Hongxun Zhang
}

Received: 9 February 2006/ Accepted: 22 April 2006/Published online: 6 June 2006

(C) Springer Science+Business Media B.V. 2006

\begin{abstract}
This study was designed to examine the reaction of microbial communities to the associated impact of inorganic fertilizers and pesticides during laboratorial simulation for 90 days. Soils were sampled from fields in three different stations of Chinese Ecosystem Research Network (CERN). The metabolic profiles were characterized by using BIOLOG GN2 microplates. The Shannon Index $(\mathrm{H})$ showed that the metabolic diversity of the three types of soil exhibited similar profiles. PCA and CLUSTER further explored the variance in community structures with the same $\mathrm{H}$. There were obvious changes in the structures of microbial communities during the 90-day duration of the work and the three different types of soil showed similar profiles for most of the period of the experiment.
\end{abstract}

Keywords Inorganic fertilizers - Pesticides ·

Metabolic diversity $\cdot$ BIOLOG

\section{Introduction}

Soil is a complex ecosystem, on which microbial communities have a great impact (Bossio and Scow 1995), and the maintenance of the productivity of soils under managed agricultural conditions is very important. In order to maintain and stimulate the growth of plants, inorganic fertilizers such as ammonium bicarbonate

J. Chen $\cdot$ X. Zhuang $(\bowtie) \cdot$ H.Xie $\cdot$ Z. Bai $\cdot$ H. Qi $\cdot$ H. Zhang

Department of Environmental Biotechnology,

Research Center for Eco-Environmental Sciences,

Chinese Academy of Sciences, P.O. Box 2871,

Beijing 100085, China

e-mails: xizhuang@mail.rcees.ac.cn; xiehuijunhb@yahoo.com.cn are regularly added. Additionally, to keep plants healthy, herbicides, pesticides and bactericides are widely used. Conflicting data have been published about the impact of inorganic fertilizers on the activities and diversity of microbial communities. Lovell et al. (1995) showed that $\mathrm{N}$ fertilizer had limited effect on soil microbial biomass, but over a long term, the activity decreased. Yang et al. (2000) reported that chemical fertilizer pollution caused an increase in the soil biomass but a decrease in the DNA diversity. Sarathchandra et al. (2001) suggested that most microbiological characteristics did not respond to fertilizer treatments. Researches on pesticides obtained the comparatively consistent conclusion that they inhibited the microbial habitats and changed their function and structure (Engelen et al. 1998; Yang et al. 2000; Thirup et al. 2001).

Microorganisms are considered to be effective bioindicators due to their capability to respond quickly to environmental changes (Avidano et al. 2005). While Wardle (1992) recommended microbial biomass as an ecological indicator of stress caused by human activities (see Zheng et al.2005), metabolic diversity is even more essential for understanding the role of microbial communities (Preston-Mafham et al. 2002). As a method for analysing metabolic diversity, BIOLOG is based on the utilization by microbial communities on different carbon sources. With results analysed by multivariate statistics such as principal component analysis (PCA) (Haack et al. 1995; Rogers and Tate III 2001) or by kinetic approaches (Lindstrom et al. 1998; Garland et al. 2001), shifts in BIOLOG metabolic diversity can be related to shifts in microbial communities.

In recent years, to ensure the productivity and quality of crops, inorganic fertilizers and pesticides 
have been consistently used in China and some other countries. Although microbial communities are significant soil bioindicators and much has been done on the microbial communities of different soils (Grayston et al. 1998; O'Donnell et al. 2001), the response of them under associated conditions is not very clear. In this study, we tried to track the associated impact of inorganic fertilizers and pesticides on microbial communities for several months and to compare the changing profiles among different soils.

\section{Materials and methods}

Sampling site

Soils in this study were sampled from three fields in the agricultural ecosystem stations of Chinese Ecosystem Research Network (CERN) of the Chinese Academy of Sciences. All these sampling sites had not been contaminated by any pesticides or fertilizers for years. The three stations represent different kinds of soils in China. Changshu (CS) station lies on Taihu Plain in the north Sub-Torrid Zone. Changwu (CW) station lies in the yellow soil area in the Temperate Zone. Luancheng (LC) station lies on Huabei Plain in the warm Temperate Zone.

In each station, three sites were randomly chosen to sample. At each site an equal amount of soils were collected from 0 to $20 \mathrm{~cm}$ depth and 20 to $40 \mathrm{~cm}$ depth separately. Then all the soils from the same station were mixed together equably and placed in plastic bags for transport to the laboratory. The soil samples were stored in the laboratory at $4^{\circ} \mathrm{C}$ for further use.

\section{Physicochemical soil properties}

The main physicochemical properties of the soils were determined by the Soil Laboratory of Beijing Forestry University. Soil $\mathrm{pH}$ was determined with a glass electrode in a 1:2.5 soil to water ratio. Organic carbon was determined by the Tiurin method. Total nitrogen was determined by the semi-micro Kjeldahl method. Available potassium was determined by flame emission photometry (Hald 1947). Readily available phosphorus was determined by colorimetric method (Zheng et al. 2005). The results are given in Table 1.

Simulation of highly intensified agricultural conditions in the laboratory

A $100 \mathrm{~g}$ soil sample was put in a flowerpot and selected kinds of pesticides and chemical fertilizers (Table 2)
Table 1 Physicochemical properties of the soils studied

\begin{tabular}{llll}
\hline Properties & CS & CW & LC \\
\hline pH & 7.79 & 8.24 & 8.23 \\
Water (\%) & 25.0 & 15.0 & 11.0 \\
Organic carbon (\%) & 2.9051 & 0.9454 & 1.1128 \\
Total nitrogen (\%) & 0.1501 & 0.0788 & 0.0815 \\
Available potassium (\%) & 0.008 & 0.011 & 0.008 \\
Readily available phosphorus (ppm) & 5.25 & 10.13 & 19.5 \\
\hline
\end{tabular}

Table 2 Pesticides and chemical fertilizers selected for the simulation

\begin{tabular}{lll}
\hline & Sort & $\begin{array}{l}\text { Quantity } \\
\left(\mathrm{mg} \mathrm{g}^{-1} \text { soil }\right)\end{array}$ \\
\hline Acetochlor & Herbicide & 5.0 \\
Fenvalerate & Pesticide & 1.0 \\
Thiophanate-Methyl & Bactericide & 2.0 \\
$\mathrm{KH}_{2} \mathrm{PO}_{4}$ & Chemical fertilizer & 0.2 \\
$\mathrm{NH}_{4} \mathrm{HCO}_{3}$ & Chemical fertilizer & 1 \\
\hline
\end{tabular}

were added for simulation of highly intensified agricultural conditions. All the flowerpots were incubated at $25^{\circ} \mathrm{C}$. Sterilized water was added in every 2 days according to the water content in Table 1 to keep constant. Soils in the flowerpots were sampled at 0,10 , 20, 40, 60 and 90 days and samples were stored at $4{ }^{\circ} \mathrm{C}$ until the further analysis. The following BIOLOG analysis was conducted within $48 \mathrm{~h}$.

\section{BIOLOG analysis}

The microbial communities were analysed by using BIOLOG GN2 microplates (Biolog, Inc., Hayward, CA). Although other plates could also be used for the analysis, GN plates have been favored by most workers (Preston-Mafham et al. 2002). Samples equivalent to $2 \mathrm{~g}$ dry soil were diluted 1000 -fold in sterile $0.85 \%$ saline solution in order to minimize the interference from the suspended and dissolved soil components. Then the samples were settled for $5 \mathrm{~min}$. Supernatant was used to inoculate the plates with $150 \mu \mathrm{l}$ each well. The microplates were then incubated at $28^{\circ} \mathrm{C}$ without agitation. The color development (absorbance at $590 \mathrm{~nm}$ ) in the wells was determined at $24 \mathrm{~h}$ intervals from 48 to $168 \mathrm{~h}$ with a microtiter-plate reader (BIOLOG System).

Data analysis

The average well color development (AWCD) was calculated to give an overall color development rate in each plate (Garland and Mills 1991). Due to the associated impact of pesticides and chemical fertilizers on 
the microbial communities, the cell inoculum density varied greatly in the plates sampled at different days during simulation, which made the results incorrect. So a set of data with fixed AWCD level was selected for univariate and multivariate analysis of metabolic diversity. A comparative low reference value was taken because the negative effect of pesticides and chemical fertilizers weakened the color development.

One perspective of examining the metabolic diversity was substrate diversity, which measures the ratio of the substrates' utilization of all the substrates on the plate. The Shannon index $(\mathrm{H})$ was calculated to designate this diversity (Zak et al. 1994).

It was very difficult to directly observe the intrinsic differences among samples with so many variables (95 substrates). So multivariate methods such as principal component analysis (PCA) and hierarchical clustering analysis (CLUSTER) were employed to determine how the samples were different as a whole. PCA could simplify the comparison by projecting the original variables onto several new axes (principal components [PCs]) (Garland and Mills 1991). The PCA and correlation analysis were performed with SPSS (version 13.0, SPSS Inc.).

CLUSTER classifies a set of samples described by numerical variables into groups (Avidano and Gamalero 2005). The similarity based on the BrayCurtis coefficient (BC), which is often a satisfactory coefficient for biological data on community structure, was calculated between every two samples (see Cheng 2004). An abundance similarity matrix was then constructed. In this study, CLUSTER was performed with Biodiversity Pro (http://www.bio.unipg.it/ecologia/download.htm).

\section{Results}

BIOLOG carbon substrate metabolic activities

The carbon substrate metabolic activities of soils from the three different stations were measured by AWCD (Fig. 1). AWCD for CS and CW soils reached highest on day 10 consistently, whereas $\mathrm{CW}$ but not CS reached the lowest on day 60 . As for LC, the highest value appeared at the beginning and the lowest on day 20 . For all the three stations the values at $168 \mathrm{~h}$ on day 90 increased greatly than at $144 \mathrm{~h}$, which might be brought in errors during measurement. As shown in Fig. 1, the AWCD varied greatly in samples from different days, so analysis of metabolic diversity was performed with a comparative low value of 0.1 .
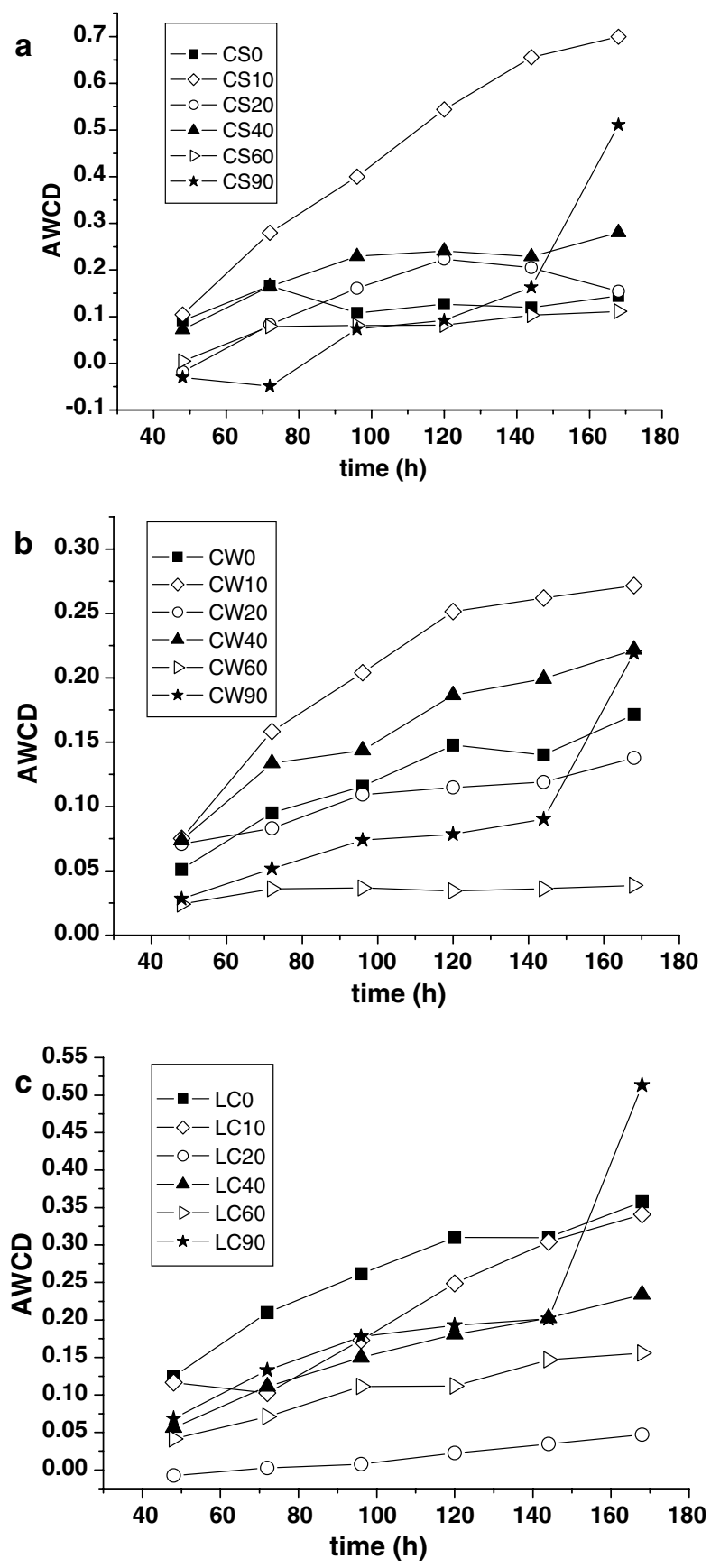

Fig. 1 Substrate utilization patterns during simulation on soils from three different stations of Chinese Ecosystem Research Network (CERN). a-c represented CS, CW and LC station, respectively, and $0,10,20,40,60$ and 90 represented sampling days during simulation

\section{Metabolic diversity patterns}

The Shannon index $(\mathrm{H})$ suggested the diversity of carbon substrates utilization by soil microbial communities during simulation. The three soils exhibited similar profiles with slight differences at the beginning 


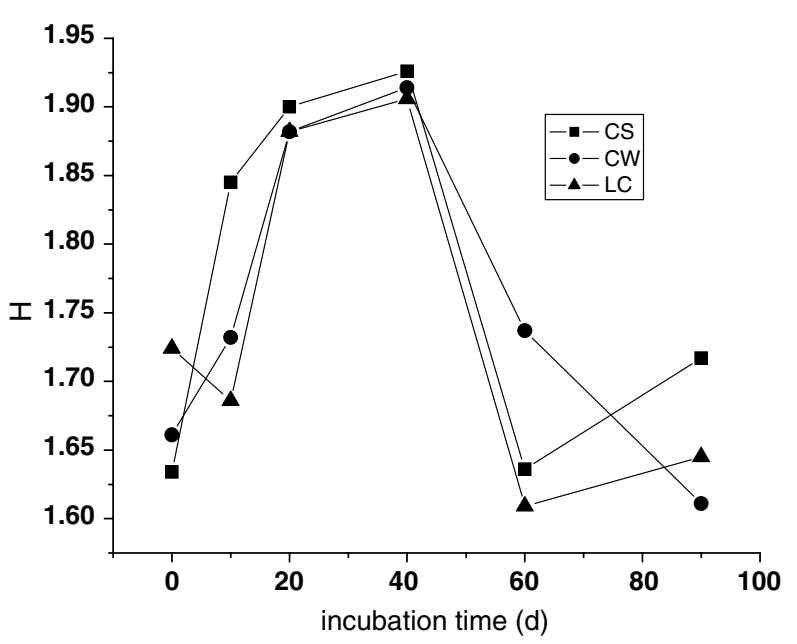

Fig. 2 Diversity patterns during simulation for soils from three different stations of Chinese Ecosystem Research Network (CERN). CS, CW and LC were the same as in Fig. 1

and the end (Fig. 2). They all got high values in the first 20 days and then declined sharply after day 40.

Measures of substrate diversity by Shannon index provided no information about the types of substrates that were assimilated by the microbial communities (Zak et al. 1994). Samples catabolizing totally different substrates might exhibit identical substrate diversity. Here although the three soils exhibited very similar substrate diversity, approaches with more information need to be employed to show which types of the substrates were utilized and the intrinsic differences between the microbial communities.

The PCA was then performed for the three soils to show the changes of the microbial communities with time during the simulation (Fig. 3). PCA for CS, with PC1 explaining $31.7 \%$ of the variance and $\mathrm{PC} 2$ of $25.8 \%$, separated samples into three groups ( 0 and 10 , 20 and 40, 60 and 90); PCA for CW, with PC1 explaining $36.5 \%$ of the variance and PC2 of $19.4 \%$, separated samples into three groups ( 0 and 40,10 and 20, 60 and 90); and PCA of LC, with PC1 explaining $27.9 \%$ of the variance and PC2 of $23.1 \%$, also separated samples also into three groups ( 0 and 10, 20 and 40, 60 and 90). The dispersion of PCs of the three soils before day 60 were different, but for each soil, the data of day 60 and day 90 gathered together and separated from the previous data.

By examining the correlation of the original variables to the PCs, the most useful carbon sources in different samples could be established. The substrates with high correlation coefficients to PCs for the three soils are shown in Tables 3-5, respectively. High correlation of a carbon source to a PC did not mean that it was highly utilized by all samples, and the carbon uti-

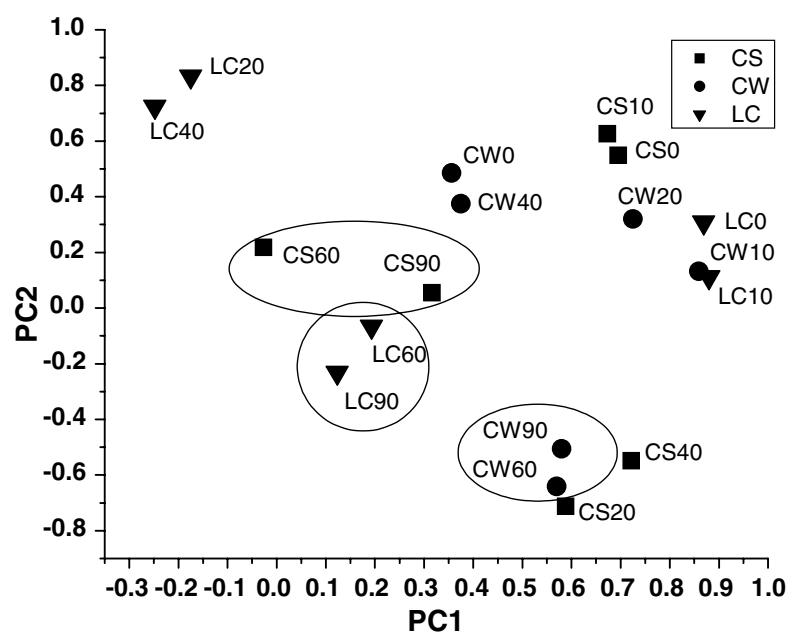

Fig. 3 Independent principal component analysis of substrate utilization patterns during simulation for soils from three different stations of Chinese Ecosystem Research Network (CERN). Sample codes were the same as in Fig. 1

lization showed great variance among the samples (Garland and Mills 1991). Substrates greatly affecting PC1 were carboxylic acids and for PC2 were carbohydrates in CS (Table 3). In CW, PC1 and PC2 were mainly affected by carbohydrates and amino acids, respectively (Table 4). Carbohydrates and carboxylic acids affected PC1 significantly while carbohydrates affected PC2 greatly in LC (Table 5).

A quantitative similarity of the microbial communities from the three soils during simulation was calculated by CLUSTER based on the Bray-Curtis coefficient (Fig. 4). Although the similarities were different among the soils, samples from day 20 and day 40 shared a high similarity in each soil. Microbial communities of day 60 and day 90 showed significant differences from the communities in soils sampled earlier as well as the results in PCA (Fig. 3).

\section{Discussion}

Sole carbon utilization pattern (BIOLOG) is a useful method for assessing functional diversity of microorganisms (Wünsche et al. 1995; Preston-Mafham et al. 2002). But due to the large amount of data, properly interpretation of a BIOLOG profile is a significant hurdle and requires a number of univariate and multivariate methods.

While the value of each well reflects the ability of soil microorganisms using certain substrate, AWCD depicts the metabolic activity as a whole. The comparison of three soils showed that AWCD fluctuated irregularly (Fig. 1), hence it was not appropriate for 
Table 3 Substrates with high correlation coefficients to PCs for samples of CS in PCA
Table 4 Substrates with high correlation coefficients to PCs for samples of $\mathrm{CW}$ in PCA

\begin{tabular}{llll}
\hline PC1 & $r$ & PC2 & $r$ \\
\hline Carbohydrates & & Carbohydrates & \\
Adonitol & 0.820 & D-Cellobiose & -0.907 \\
L-Arabinose & 0.908 & i-Erythritol & -0.878 \\
L-Fucose & -0.851 & D-Psicose & -0.906 \\
Carboxylic acids & & D-Raffinose & -0.822 \\
cis-Aconitic acid & -0.820 & L-Rhamnose & -0.816 \\
Pyruvic acid methyl ester & -0.769 & D-Trehalose & -0.852 \\
D-Glucosaminic acid & -0.797 & Carboxylic acids & -0.938 \\
$\beta$-Hydroxybutyric acid & 0.702 & Formic acid & -0.834 \\
Itaconic acid & -0.741 & D-Galacturonic acid & -0.909 \\
Amino acids & & D-Glucuronic acid & -0.857 \\
L-Glutamic acid & -0.864 & $\gamma$-Hydroxybutyric acid & \\
Hydroxy-L-proline & 0.774 & Amino acids & -0.857 \\
L-Serine & 0.700 & D,L-Carnitine & \\
Brominated chemicals & & Aromatic chemicals & -0.962 \\
Bromosuccinic acid & 0.827 & Thymidine & \\
Phosphorylated compounds & & Amines & -0.897 \\
$\alpha$-D-Glucose-1-Phosphate & 0.848 & Phenylethylamine & \\
& & Alcohols & \\
& & 2,3-Butanediol & -0.876 \\
& &
\end{tabular}

\begin{tabular}{lllr}
\hline PC1 & $r$ & PC2 & $r$ \\
\hline Carbohydrates & & Carbohydrates & \\
$N$-Acetyl-D-glucosamine & 0.777 & D-Arabitol & 0.869 \\
$\alpha$-D-Glucose & 0.669 & D-Galactose & 0.873 \\
D-Sorbitol & -0.682 & Turanose & 0.829 \\
D-Trehalose & -0.630 & Carboxylic acids & \\
Carboxylic acids & & D-Glucosaminic acid & 0.780 \\
$\begin{array}{l}\alpha \text {-Ketoglutaric acid } \\
\text { D-Saccharic acid }\end{array}$ & 0.805 & Amino acids & \\
Amino acids & 0.689 & L-Alanine & 0.769 \\
L-Glutamic acid & & L-Aspartic acid & -0.855 \\
Aromatic chemicals & 0.824 & L-Proline & 0.883 \\
Urocanic acid & & L-Pyroglutamic acid & 0.816 \\
Amines & 0.839 & L-Serine & 0.869 \\
Putrescine & & & \\
Phosphorylated compounds & 0.792 & & \\
D-Glucose-6-Phosphate & -0.818 & & \\
\hline
\end{tabular}

use directly for community-level analysis. Garland and Mills (1991) concluded that optimal characterization of BIOLOG requires approximately equivalent inoculum density. But this is not practical for analysing soil samples, because cell number counts or estimate of microorganisms delays the process and the communities are likely to change during this time (PrestonMafham et al. 2002). Additionally, the same sample with different AWCD showed variance in the univariate and multivariate analysis, which indicated that microbial communities might change during measurement (data not shown). A means of compensating for differences in inoculum density and reflecting the real variance is to use a fixed level of AWCD to determine the data for further analysis (Garland 1996).

Although Shannon Indices $(\mathrm{H})$ of all the three soils increased greatly in the first 20 days (Fig. 2), the changing of the metabolic activities was quite different (Fig. 1), which suggested that the impact of inorganic fertilizers and pesticides on microbial communities in the three soils were different at the early stage. After day $40, \mathrm{H}$ decreased to be approximately the same on day 90 as at the beginning (Fig. 2). But the multivariate methods of PCA and CLSTER both showed that there existed obvious variance between microbial communities at the beginning and end (Figs. 3, 4), which suggested that impact of inorganic fertilizers and pesticides on microbial communities of different soils may have similar mechanisms, which indicated that the associated impact probably caused changing in the community structure even with the same H. Although shifts in microbial communities could be reflected by the shifts in BIOLOG metabolic diversity (Haack et al. 1995), appropriate statistical 
Table 5 Substrates with high correlation coefficients to PCs for samples of LC in PCA

\begin{tabular}{lrlr}
\hline PC1 & $r$ & PC2 & $r$ \\
\hline Carbohydrates & & Carbohydrates & \\
$N$-Acetyl-D-glucosamine & 0.804 & meso-Inositol & 0.904 \\
L-Arabinose & -0.707 & Maltose & 0.909 \\
Meso-Inositol & -0.737 & D-Melibiose & 0.844 \\
Maltose & -0.707 & D-Raffinose & 0.870 \\
D-Raffinose & -0.764 & Xylitol & 0.842 \\
Carboxylic acids & & Adonitol & 0.776 \\
Pyruvic acid methyl ester & 0.730 & L-Arabinose & 0.750 \\
D-Glucuronic acid & -0.740 & D-Arabitol & 0.723 \\
Itaconic acid & -0.711 & a-D-Lactose & 0.719 \\
D-Galactonic acid Lactone & 0.883 & Lactulose & 0.750 \\
$\alpha$-Ketoglutaric acid & 0.935 & L-Rhamnose & 0.725 \\
Amino acids & & Carboxylic acids & \\
L-Histidine & -0.802 & D-Glucuronic acid & 0.910 \\
L-Leucine & 0.908 & p-Hydroxyphenylacetic acid & 0.714 \\
Alcohols & & Amino acids & \\
Glycerol & 0.858 & L-Aspartic acid & -0.780 \\
Phosphorylated compounds & & L-Histidine & 0.838 \\
D-Glucose-6-Phosphate & -0.843 & Amines & \\
& & Phenylethylamine & 0.826 \\
& & Alcohols & \\
& & 2,3-Butanediol & 0.706 \\
\hline
\end{tabular}

method were also required to explore the intrinsic difference of community structures from the crude data.

In recent years, researches have been performed on soils contaminated with fertilizers or pesticides for many years (Yang et al. 2000; Thirup et al. 2001; Sarathchandra et al. 2001). Soils were sampled directly from land at one or a few time points. In our research, simulation of associated impact of inorganic fertilizers and pesticides was performed in laboratory. The process of these factors on soil microbial communities could be fully monitored during simulation and disturbance such as plants or seasonal effect could be avoided. With many other factors such as temperature and water content constant, the results reflected exactly the effect of anthropic activities to microbial communities in soils.

Along with the development of molecular biology, a number of molecular methods such as random amplified polymorphic DNA (RAPD) and denaturing gradient gel electrophoresis (DGGE) have been applied in microbial diversity (Yang et al. 2000; Rölleke et al. 1999). These techniques could characterize the microbial communities more accurately on the molecular scale, but the relationship between the genetic diversity and metabolic diversity and whether the genetic diversity could reflect community-level interactions are uncertain. We have noticed that although BIOLOG was a method characterizing cultivable heterotrophic bacterial communities and the data obtained from it did not represent the whole communities 
(Preston-Mafham et al. 2002; Nannipieri et al. 2003), it provided much more information on the communitylevel.

In conclusion, our study showed that the structure of microbial communities exhibited obvious variance after treatment with inorganic fertilizers and pesticides in the laboratory and the three different soils showed similar profiles for a long period. But the exact change in the microbial communities is not clear and remains an important issue to be explored.

Acknowledgements This work was supported by the National Science Foundation of China (NSFC, Project No. 30470289 and 20477051).

\section{References}

Avidano L, Gamalero E, Cossa GP, Carraro E (2005) Characterization of soil health in an Italian polluted site by using microorganisms as bioindicators. Appl Soil Ecol 30:21-33

Bossio DA, Scow KM (1995) Impact of carbon and flooding on the metabolic diversity of microbial communities in soils. Appl Environ Microbiol 61:4043-4050

Cheng CC (2004) Statistical approaches on discriminating spatial variation of species diversity. Bot Bull Acad Sinica 45:339_ 346

Engelen B, Meinken K, Von Wintzingerode F, Heuer H, Malkomes HP, Backhaus H (1998) Monitoring impact of a pesticide treatment on bacterial soil communities by metabolic and genetic fingerprinting in addition to conventional testing procedures. Appl Environ Microbiol 64:2814-2821

Garland JL (1996) Analytical approaches to the characterization of samples of microbial communities using patterns of potential C source utilization. Soil Biol Biochem 28:213-221

Garland JL, Mills AL (1991) Classification and characterization of heterotrophic microbial communities on the basis of patterns of community-level sole-carbon-source utilization. Appl Environ Microbiol 57:2351-2359

Garland JL, Mills AL, Young JS (2001) Relative effectiveness of kinetic analysis vs single point readings for classifying environmental samples based on community-level physiological profiles (CLPP). Soil Biol Biochem 33:1059-1066

Grayston SJ, Wang SQ, Campbell CD, Edwards AC (1998) Selective influence of plant species on microbial diversity in the rhizosphere. Soil Biol Biochem 30:369-378

Haack SK, Garchow HM, Klug J, Forney LJ (1995) Analysis of factors affecting the accuracy, reproducibility, and interpretation of microbial community carbon source utilization patterns. Appl Environ Microbiol 61:1458-1468
Hald PM (1947) The flame photometer for the measurement of sodium and potassium in biological materials. J Biol Chem 167:499-510

Lindstrom JE, Barry RP, Braddock JF (1998) Microbial community analysis: a kinetic approach to constructing potential C source utilization patterns. Soil Biol Biochem 30:231-239

Lovell RD, Jarvis SC, Bardgett RD (1995) Soil microbial biomass and activity in long-term grassland: effects of management change. Soil Biol Biochem 27:969-975

Nannipieri P, Ascher J, Ceccherini MT, Landi L, Pietramellara G, Renella G (2003) Microbial diversity and soil functions. Eur J Soil Sci 54:655-670

O'Donnell AG, Seasman M, Macrae A, Waite I, Davies JT (2001) Plants and fertilisers as drivers of change in microbial community structure and function in soils. Plant Soil 232:135-145

Preston-Mafham J, Boddy L, Randerson PF (2002) Analysis of microbial community functional diversity using sole-carbonsource utilization profiles-a critique. FEMS Microbiol Ecol 42:1-14

Rogers BF, Tate RL III (2001) Temporal analysis of the soil microbial community along a toposequence in Pineland soils. Soil Biol Biochem 33:1389-1401

Rölleke S, Gurter C, Drevello U, Drewello R, Lubitz W, Weissmann R (1999) Analysis of bacterial communities on historical glass by denaturing gradient gel electrophoresis of PCR-amplified gene fragments coding for 16S rRNA. J Microbiol Methods 36:107-114

Sarathchandra SU, Ghani A, Yeates GW, Butch G, Cox NR (2001) Effect of nitrogen and phosphate fertilizers on microbial and nematode diversity in pasture soils. Soil Biol Biochem 33:953-964

Thirup L, Johnsen K, Torsvik V, Spliid NH, Jacobsen CS (2001) Effects of fenpropimorph on bacteria and fungi during decomposition of barley roots. Soil Biol Biochem 33:15171524

Wardle DA (1992) A comparative assessment of factors which influence microbial biomass carbon and nitrogen levels in soil. Biol Rev Camb Philos Soc 67:321-358

Wünsche L, Brüggemann L, Babel W (1995) Determination of substrate utilization patterns of soil microbial communities: an approach to assess population changes after hydrocarbon pollution. FEMS Microbiol Ecol 17:295-306

Yang YH, Yao J, Hu S, Qi Y. (2000) Effects of agricultural chemicals on DNA sequence diversity of soil microbial community: a study with RAPD marker. Microb Ecol 39:72-79

Zak JC, Willing MR, Moorhead DL, Wildman HG (1994) Functional diversity of microbial communities: a quantitative approach. Soil Biol Biochem 26:1101-1108

Zheng H, Ouyang ZK, Wang XK, Fang ZG, Zhao TQ, Miao H (2005) Effects of regenerating forest cover on soil microbial communities: a case study in hilly red soil region, Southern China. For Ecol Manage 217:244-254 